MICHAEL LEINERT

\title{
Some aspects of Segal Algebras
}

\author{
Estratto dai
}

«Rendiconti del Seminario Matematico e Fisico di Milano» Vol. XLV (1975)

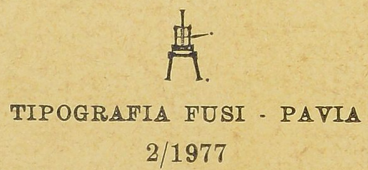



MICHAEL LEINERT

\section{Some aspects of Segal Algebras}

Estratto dai

«Rendiconti del Seminario Matematico e Fisico di Milano»

Vol. XLV (1975)

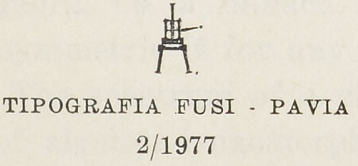



MICHAEL LEINERT

dell'Università di Heidelberg

\section{SOME ASPECTS OF SEGAL ALGEBRAS}

(Conferenza tenuta il 9 aprile 1975)

Sunto. - Le «Algebre di Segal» sono ideali densi nell'algebra di gruppo. I risultati, riportati in questa nota, mettono in luce alcuni comportamenti che in qualche senso rendono simili (vedi paragrafo I), o differenziano (paragrafo II), queste algebre, dall'algebra di gruppo.

La maggior parte dei risultati riportati può essere trovata in [1]; $\ldots$ [7].

Segal algebras are dense ideals in group algebras. They behave in some sense like group algebras (see section I), but they also are quite different in some other sense (section II). Most of the results mentioned below can be found in [1]-[7].

$G$ denotes a locally compact group, $C(G)$ the space of bounded continuous functions on $G, C_{0}(G)$ the space of continuous functions vanishing at infinity, $L^{p}(G), 1 \leq p \leq \infty$, the usual $L^{p}$ spaces with respect to some left Haar measure $d x$ on $G$. If $G$ is commutative we denote by $\widehat{G}$ its dual group and write $\widehat{f}$ for the Fourier transform of a function $f \in L^{1}(G)$. If $A$ is a Banach algebra, $M(\$)$ is the space of left multipliers i.e. of bounded linear operators $T$ on $\$$ with $T(a b)=(T a) b$ for all $a, b \in \$$. The closure of a subset $J \notin A$ is $\bar{J}^{A}$. We say that $A$ factorizes if any $a \in A$ can be written $a=b \cdot c$ with $b, c \in A$. $A$ has right approximate units, if for any $a \in A$ and $\varepsilon>0$ there is some $u \in A$ such that $|a-a u|_{A}<\varepsilon$; if there is a constant $K>0$ such that $u$ can be chosen with norm $|u|_{A} \leq K$, then $A$ is said to have bounded right approximate units. It is clear what will be meant by left approximate and bounded left approximate units. If $A$ is a Banach-*-algebra, i.e. a Banach algebra with isometric involution, $A$ is called symmetric if for any $a \in A$ the element $a * a$ has positive spectrum. The spectrum of a commutative Banach algebra $B$ is the space of algebra homomorphisms from $B$ onto the 
complex numbers. All algebras and vector spaces under consideration are complex.

DEFINITION 1. - Let $G \frac{b^{\frac{b}{a}}}{a}$ locally compact group. A Segal algebra $S$ in $L^{1}(G)$ is a dense linear subspace of $L^{1}(G)$ which is a Banach space under some norme ||$_{s}$ and satisfies:

(1) There is some $D>0$ such that $|f|_{S} \geq D|f|_{1}$ for all $f \in S$

(2) If for $f \in S$ and $x \in G$ we define ${ }_{x} f$ by ${ }_{x} f(y)=f\left(x^{-1} y\right)$ for $y \in G$, then ${ }_{x} f \in S$ for all $f \in S, x \in G$, and there is some $C>0$ such that

$$
\left|{ }_{x} f\right|_{S} \leq C|f|_{s} \text { for all } x \in G .
$$

(3) For $f \in S$ the mapping $x \mapsto{ }_{x} f$ is continuous from $G$ to $S$ (it suffices to require continuity at the identity of $G$ ).

By vectorvalued integration it follows that $S$ is a left ideal in $L^{1}(G)$ and

$$
|f * g|_{S} \leq C|f|_{1}|g|_{S} \text { for } f \in L^{1}(G), g \in S
$$

in particular

$$
|f * g|_{S} \leq C \cdot D^{-1}|f|_{S}|g|_{S} \text { for } f, g \in S .
$$

So, in an equivalent norm, $S$ is a Banach algebra.

Definition 2. - Let $A$ be a Banach algebra. An abstract Segal algebra (or: left normed ideal) in $A$ is a dense left ideal $B$ of $A$ which is a Banach space under some norm ||$_{B}$ and satisfies:

(6) There is some $D>0$ such that

$$
|b|_{B} \geq D|b|_{A} \text { for all } b \in B
$$

(7) There is some $C>0$ such that

$$
|a b|_{B} \underset{L}{ } C|a|_{A}|b|_{B} \text { for all } a \in A, b \in B \text {. }
$$

This last inequality is automatic and follows from the preceding assumptions.

Obviously, any Segal algebra in a group algebra $L^{1}(G)$ is a left normed ideal in $L^{1}(G)$; it always has left approximate units.

Conversely a left normed ideal in $L^{1}(G)$ having left approximate units is a Segal algebra. This follows by means of (7) and the Cohen factcrization theorem. 
EXAMPLES. - a) Let $G$ be the circle group and $S$ be $C(G)$ with the usual norm. $S$ is a Segal algebra in $L^{1}(G)$.

b) Let $G$ be the circle group and $B$ be $L^{\infty}(G)$. Then $B$ is a left normed ideal in $L^{1}(G)$, but it is not a Segal algebra, since translation is not continuous.

c) Let $G$ be the circle group and $S$ be $L^{p}(G), 1<p<\infty$. Then $S$ is a Segal algebra in $L^{1}(G)$.

d) For $G$ locally compact abelian and $1 \leq p<\infty$ let

$$
S=\left\{f \in L^{1}(G) \mid \widehat{f} \in L^{p}(\widehat{G})\right\} \text { with norm }|f|_{S}=|f|_{1}+|\widehat{f}|_{p} .
$$

Then $S$ is a Segal algebra in $L^{1}(G)$.

e) For $G$ locally compact and $1<p<\infty$ let $S=L^{1}(G) \cap L^{p}(G)$ with norm $|f|_{S}=|f|_{1}+|f|_{p}$. Then $S$ is a Segal algebra in $L^{1}(G)$.

f) For $G$ locally compact let $S=L^{1}(G) \cap C_{0}(G)$ with norm $|f|_{S}=$ $=|f|_{1}+|f|_{\infty}$. Then $S$ is a Segal algebra. The algebra $B=$ $=L^{1}(G) \cap C(G)$ is a left normed ideal in $L^{1}(G)$.

g) Let $A$ be the Banach algebra of compact operators on infinite dimensional Hilbert space and let $B$ be the subspace of HilbertSchmidt operators with the Hilbert-Schmidt norm. $B$ is a left normed ideal in $A$.

I.

Theorem 3. - a) Let $A$ be a Banach algebra and $B$ be a left normed ideal in $A$, and suppose that $A$ and $B$ have right approximate units. If $I$ is a closed right (two-sided) ideal in $A$, then $I \cap B$ is a closed right (two-sided) ideal in $B$, and the mapping $I \mapsto I \cap B$ is bijective from the set of closed right (two-sided) ideals in $A$ onto the set of closed right (two-sided) idealts in $B$. The inverse mapping is given by $J \mapsto \bar{J}^{A}$.

b) For maximal modular left ideals the corresponding statement holds without the assumption on $A$ and $B$ to have approximate units.

It is not known if part $a$ ) of the above theorem can be extended to closed left ideals.

COROLlary 1. - If $A$ is semi-simple, so is $B$. The converse holds if and only if $A$ satisfies $\{a \in A \mid a A=0\}=\{0\}$. 
Corollary 2. - If $A$ is a Banach-*-algebra and $B$ is a left normed ideal in $A$ with $B^{*}=B$, then $B$ is symmetric if and only if $A$ is.

Proposition 4. - Let $A$ be a Banach algebra and $B$ a left normed ideal in $A$. For any strictly irreducible vector space representation $\pi$ of $A$, the restriction $\left.\pi\right|_{B}$ is a strictly irreducible representation of $B$, and the mapping $\left.\pi \mapsto \pi\right|_{B}$ is bijective from the strictly irreducible representations of $A$ onto those of $B$.

COROLLARY. - If $G$ is a locally compact abelian group and $S$ is a $\checkmark$ in Segal algebra $L^{1}(G)$, then the spectrum of $S$ is equal to the spectrum of $L^{1}(G)$, hence to $\widehat{G}$. From Theorem 3 we obtain that a closed subset of $\widehat{G}$ is of spectral synthesis for $L^{1}(G)$ if and only if it is of spectral synthesis for $S$.

Proposition 5. - Let $A$ be a Banach-* algebra and $B$ a left normed ideal in $A$ with $B^{*}=B$. The restriction mapping $\left.\pi \mapsto \pi\right|_{B}$ is bijective from the Hilbert space representations of $A$ onto those of $B$.

\section{II.}

Right approximate units in left normed ideals are always unbounded:

Proposition 6. - Let $A$ be a Banach algebra and $B$ a left normed ideal in $A$. If $B$ has bounded right approximate units, then $B=A$.

Left normed ideals may factorize, they may even have a left unit (though they may not have a right unit as we have just seen), but it is not known if a Segal algebra can factorize. A partial result is

Proposition 7. - Let $G$ be a locally compact nondiscrete abelian group with dual group $\widehat{G}$. Suppose $\widehat{G}$ to be compactly generated and let $U=U^{-1}$ be a generating compact subset of $\widehat{G}$. Let $S$ be a Segal algebra in $L^{1}(G)$ with $\widehat{S}=\{\widehat{f} \mid f \in S\} \subset L^{p}(\widehat{G})$ for some finite $p$. Let $f \neq 0 \in S$ be such that all translates of $\widehat{f}$ by elements of $\widehat{G}$ are in $\widehat{S}$. If there is a real polynomial $P$ such that

$$
\left|a_{a} \widehat{f}\right| \widehat{s} \leq P(n)
$$

for all $a \in U^{n}$ and all $n \in N$, then $S$ does not factorize. 
Segal algebras may have more left multipliers than the corresponding $L^{1}$-algebras. If $G$ is a locally compact group and $S$ a Segal algebra in $L^{1}(G)$, any operator $T \in M\left(L^{1}(G)\right.$ ) leaves $S$ invariant, so that $\left.T\right|_{S}$ is in $M(S)$, but the map $\left.T \mapsto T\right|_{S}$ need not be surjective, as is seen from the following example: Let $G$ be the circle group. Then $S=L^{2}(G)$ (with its usual norm) is a Segal algebra in $L^{1}(G)$. Any $f \in L^{\infty}(G)=l^{\infty}$ defines by Fourier transformation some $T_{f} \in M(S)$, but only functions that are transforms of bounded measures have $T_{f} \in M\left(L^{1}(G)\right)$.

Summary. - Segal algebras are dense ideals in group algebras. The results, reported below, point out that Segal algebras behave in some sense like group algebras (see section I), but they also are quite different in some other sense (section II).

\section{R E F E R E N C E S}

[1] BARNES B. A., Banach algebras which are ideals in a Banach algebra. Pacific J. Math. 38 (1971), 1-7.

[2] Burnham J. T., Closed ideals in subalgebras of Banach algebras I. Proc. Amer. Math. Soc. 32 (1972), 551-555.

[3] Dunford D. H., Segal algebras and left normed ideals. J. London Math. Soc. (2) 8 (1974), 514-516.

[4] Feichtinger H. G., Zur Idealtheorie von Segal-Algebren. Manuscripta math. 10 (1973), 307-312.

[5] Leinert M., A contribution to Segal algebras. Manuscripta math. 10 (1973), 297-306.

[6] Leinert M., Remarks on Segal algebras, to appear. (Manuscripta math.).

[7] Reiter H., L1-Algebras and Segal Algebras. Lecture Notes in Mathematics 231, Springer 1971. 


